\section{Cardiovascular Response to Qigong Training in European and Asian Participants}

\section{Suphannika Ladawan ${ }^{1 *}$, Naruemon Leelayuwat ${ }^{2,3}$, Panaka- porn Wannanon ${ }^{2,3}$, Kultida Klarod ${ }^{4}$, Verena Menz ${ }^{1}$, Hannes Gatterer $^{1}$ and Martin Burtscher ${ }^{1}$}

${ }^{1}$ Department of Sport Science, Medical Section, Faculty of Psychology and Sport Science, University of Innsbruck, Innsbruck, Austria

${ }^{2}$ Department of Physiology, Faculty of Medicine, Khon Kaen University, Khon Kaen, Thailand

${ }^{3}$ Exercise and Sport Sciences Development and Research Group, Khon Kaen University, Khon Kaen, Thailand

${ }^{4}$ Department of Physical Therapy, Faculty of Allied Health Sciences, Burapha University, Chon Buri, Thailand

\begin{abstract}
Background: Qigong exercise has been shown to improve cardiovascular function in clinical populations. Ethnicity differences in cardiovascular responses to Qigong exercise have not been studied extensively, especially regarding females. Thus, the purpose of this study was to compare cardiovascular effects to Qigong training between healthy, sedentary Asian and European female participants.

Methods: In this study, European and Asian participants were lifelong European and Asian residents respectively. Six healthy, sedentary Asian females (aged 53.0 \pm 5.7 years) and 6 European healthy, sedentary females (aged 49.6 \pm 7.4 years) performed Qigong exercise 3 days per week for 8 weeks followed by 12 weeks of no exercise. Blood pressure and heart rate values at rest and during submaximal exercise were assessed at baseline (T1), after the end of the training program (T2), and after 12 weeks of exercise cessation (T3).

Results: European participants showed a significant reduction in Systolic Blood Pressure (SBP) and Mean Arterial Pressure (MAP) after Qigong training (SBP: T=130.83 $\pm 18.82 \mathrm{mmHg}, \mathrm{T} 2=111.83 \pm 9.09$ $\mathrm{mmHg}, \mathrm{p}=0.05$; MAP: $\mathrm{T} 1=96.94 \pm 6.95 \mathrm{mmHg}, \mathrm{T} 2=87.95 \pm 6.00 \mathrm{mmHg}$, $p=0.02$ ), which returned to baseline level after exercise cessation
\end{abstract}

*Corresponding author: Suphannika Ladawan, Department of Sport Science, Medical Section, Faculty of Psychology and Sport Science, University of Innsbruck, Innsbruck, Austria, Tel: +43 051250745896; E-mail: suphannika.ladawan@student.uibk.ac.at; Gehpt18@gmail.com

Citation: Ladawan S, Leelayuwat N, Wannanon P, Klarod K, Menz V, et al. (2017) Cardiovascular Response to Qigong Training in European and Asian Participants. J Altern Complement Integr Med 3: 040.

Received: September 13, 2017; Accepted: October 12, 2017; Published: October 27, 2017
(SBP: T3=124.17 $\pm 10.21 \mathrm{mmHg}, \quad p=0.01 ; \quad$ MAP: $\quad T 3=96.94 \pm 4.77$ $\mathrm{mmHg}, \mathrm{p}=0.01$ ). Asian participants showed slightly reduced SBP values after the training but these changes did not reach statistical significance $(\mathrm{T} 1=123.00 \pm 13.48 \mathrm{mmHg}, \mathrm{T} 2=116.83 \pm 6.46 \mathrm{mmHg}$, $p=0.22$ ). Heart rates at submaximal exercise was reduced after training in both groups (European participants: $T 1=118.67 \pm 9.48$ beats $/ \mathrm{min}, \mathrm{T} 2=112.83 \pm 7.78$ beats $/ \mathrm{min}, \mathrm{p}=0.03$; Asian participants: $\mathrm{T} 1=113.50 \pm 4.32$ beats $/ \mathrm{min}, \mathrm{T} 2=101.83 \pm 11.87$ beats $/ \mathrm{min}, \mathrm{p}<0.01)$. No statistically significant differences were observed between groups.

Discussion: Qigong training resulted in lower heart rates to submaximal exercise in both populations and lower blood pressure values especially in people with higher values at baseline (European participants). However, beneficial effects on blood pressure disappeared after 12 weeks of exercise cessation. Therefore, training continuation is of essential importance. Further research is required to examine whether our SBP findings in response to Qigong training were due to higher baseline blood pressure, sample bias (migration) or ethnicity differences.

Keywords: Cardiovascular function; Ethnicity; Qigong

\section{Introduction}

Qigong is ancient chinese exercise including movement focusing on breathing, a meditative state of mind, and relaxation [1]. Focus on breathing during Qigong practice may bring the mind to a restful state and oxygenation and/or energy to the body [1]. Altering the breathing pattern may be associated with changing of the autonomic nervous system function [1,2]. Qigong exercise has been shown to have beneficial effects on cardiovascular function such as improved blood pressure, heart rate variability and peripheral vasomotor responses [3-6]. Tai Chi Qigong Shibashi or Tai Chi Qigong 18 forms is a set of Qigong exercises which synchronize the gentle 18 movements with breathing exercise and relaxing [7]. It is one of the most popular forms of Qigong because the movements are not complex, easy to learn, with low impact on joints, so it is suitable for people of all ages.

Qigong is widely practiced in Asia and now also becoming popular in Europe. Previous studies reported that Caucasians had higher mean total lung capacity, vital capacity, fat free mass, inspiratory and expiratory muscle pressures and wider chests than Chinese and Indians [8]. Korotzer et al., reported that Asians have lower forced vital capacity, Forced Expiratory Volume in 1 second (FEV1), and alveolar volume than Europeans [9]. Although, it is clear that ethnicity is different in physical appearance, ethnicity differences in cardiovascular responses to exercise are not well understood. Bouchard and Rankinen suggested that race was not an important factor influencing training responses [10]. However, Swift et al., found that African American women have lower cardiorespiratory fitness at baseline and an attenuated the response to aerobic exercise training compared with Caucasian American women [11]. Therefore, the present study aimed at comparing cardiovascular responses to Qigong training between healthy, sedentary European and Asian participants. This study focus on healthy, sedentary participants because there are only few studies investigate effect of Qigong exercise in healthy sedentary. In addition, previous studies reported that Qigong was light to moderate intensity 
exercise $[12,13]$. This exercise may be alternative exercise for sedentary people to start regular exercise.

\section{Materials and Methods}

\section{Participants}

To estimate the sample size, the alpha level and power of test were set at 0.05 and 0.80 respectively. Mean and SD were refer to previous study that investigated Qigong reduced blood pressure and catecholamine levels of patients with essential hypertension [14]. The calculated sample size was 4 . Due to a small sample size, 2 participants were added in each group. Six healthy, sedentary European females were recruited from Innsbruck, Austria by email and flyers, and 6 healthy, sedentary Asian females were recruited from KhonKaen, Thailand by face to face contact, and flyers. All of them were 45 and 60 years old, and did not perform regular daily exercise and did not take part in training at an athletic level at least 3 months prior to enrolling for the study. Exclusion criteria were any medical condition, such as cardiovascular diseases, respiratory diseases, orthopedic problems, neurological disorders and infectious conditions, not being compatible with performing Qigong exercise safely. They were also asked to maintain their usual activities of daily living and dietary behavior throughout the study period. Before signing the consent form for participation, participants were informed about the experimental protocol and possible risks. Ethical approval was obtained from the review board of the institute of sport science and the ethics committee of the University of Innsbruck, Austria, protocol number 11-2015, and the ethical committee of Khon Kaen University, Thailand, reference number HE591225.

\section{Study protocol}

The present study has been divided into 2 parts: the first part examined the effects of Qigong exercise on cardiovascular function. Before the first Qigong session, all participants were asked to complete the baseline measurements (T1), then they performed Qigong exercise for 8 weeks followed by the first post-test (T2) after the end of the training program. The second part evaluated the effects after cessation of Qigong exercise in subjects who volunteered to return to previous habits and did not perform Qigong exercise for 12 weeks (T3). Qigong training and data collection were conducted in Innsbruck, Austria for the European group, and in Khon Kaen, Thailand for Asian group.

\section{Qigong exercise}

All participants were required to performed Qigong 18 forms or Tai Chi Qigong Shibashi 3 days a week for 8 weeks. The movements of Qigong were adapted from 18 movements Taiji Qigong by Robinson [15]. The exercise started with 10 minutes warm up by general stretching. After that, they performed 18 movements Qigong exercise (Tai Chi Qigong Shibashi) for 40 minutes followed by cool down with general stretching for 10 minutes. Both Asian and European participant groups, Qigong exercise was demonstrated and supervised by the same researcher, who has practiced Qigong for more than ten years.

\section{Measurements}

Cardiovascular function, blood pressure and heart rate, were measured in the afternoon (between 1 - $6 \mathrm{pm}$ ) only having a light meal at least 2 hours prior to testing. All participants were asked to avoid caffeine, alcohol intake, and smoking at least two hours prior to the investigation. Heart Rate (HR), Systolic Blood Pressure (SBP) and Diastolic Blood Pressure (DBP) were measured in a seated position after a minimum of 5 minutes rest. Mean Arterial Pressure (MAP) was calculated by MAP $=[(2 \mathrm{x}$ diastolic $)+$ systolic $] / 3$.

Submaximal exercise HR was obtained by HR during incremental cycling exercise test. The exercise began with warming up at 0 watt for 1 minute, and then workloads were increased to 25 watts for 2 minutes. After that, the workload was increased to 50 watts for 2 minutes. A 3-lead ECG was recorded throughout the test. HR at the end of 2 minutes of 50 watts was defined as submaximal HR.

\section{Statistical analysis}

Shapiro-Wilk test was used to assess the distribution of the results for each parameter. Differences between the two groups were compared using independent sample t-test for parametric or Mann Whitney U test for non-parametric data. Differences between T1, T2 and T3 within each group were compared using repeated-measures Analysis of Variance (ANOVA) with the Least Significant Difference (LSD) post hoc test. A $p$ value of $\leq 0.05$ was considered statistically significant. All data analysis was conducted with SPSS version 17.

\section{Results}

There was no participant's dropout in both groups. European and Asian participants attended $77.79 \%$ (18.67 \pm 3.78 sessions) and 82.63 $\%$ (19.83 \pm 3.25 sessions) of 24 sessions during 8 weeks of Qigong exercise program, respectively. All of them did not perform Qigong during 12 weeks of detraining phase. Baseline characteristics of participants were shown in table 1. European participants were taller than Asian participants but Body Mass Index (BMI) was not significant difference.

\begin{tabular}{|c|c|c|}
\hline \multicolumn{4}{|c|}{ Characteristic } & European (n=6) & Asian (n=6) \\
\hline Age & $49.6 \pm 7.4$ & $53.0 \pm 5.7$ \\
\hline Height $(\mathrm{cm})$ & $165.0 \pm 4.0$ & $151.0 \pm 0.0 *$ \\
\hline Body mass $(\mathrm{kg})$ & $60.0 \pm 8.1$ & $55.5 \pm 6.51$ \\
\hline BMI $\left(\mathrm{kg} / \mathrm{m}^{2}\right)$ & $22.0 \pm 2.0$ & $24.5 \pm 3.3$ \\
\hline$\%$ Body fat & $34.0 \pm 5.0$ & $33.7 \pm 3.7$ \\
\hline \multicolumn{3}{|c|}{ Table 1: Baseline characteristics. }
\end{tabular}

Data are represented as mean $\pm \mathrm{SD}$; significant difference $\mathrm{p}<0.05$.

After 8 weeks of Qigong, European participants showed significant lowering in $\operatorname{SBP}(\mathrm{p}=0.02)$ and MAP $(\mathrm{p}=0.05)$ which returned to baseline after 12 weeks of Qigong cessation (SBP; $\mathrm{p}=0.01$, and MAP; $\mathrm{p}=0.01$ ). DBP was not changed after Qigong training but increased significantly after 12 weeks cessation of training $(\mathrm{p}=0.004)$. Asian participants tended to decrease SBP after the Qigong training but the change did not reach statistical significance $(p=0.22)$. Resting heart rates remained unchanged within both groups from $\mathrm{T} 1$ to $\mathrm{T} 2$ and to T3 (Table 2 and Figure 1). No significantly different changes were observed between groups.

Compared to before training, submaximal exercise heart rate decreased significantly after 8 weeks of Qigong training in both Asian and European participants (Table 3). 
Citation: Ladawan S, Leelayuwat N, Wannanon P, Klarod K, Menz V, et al. (2017) Cardiovascular Response to Qigong Training in European and Asian Participants. J Altern Complement Integr Med 3: 040.

\begin{tabular}{|c|c|c|c|}
\hline \multirow{2}{*}{ Variables } & Time & European $(\mathbf{n}=\mathbf{6})$ & Asian $(\mathbf{n}=\mathbf{6})$ \\
\hline \multirow{3}{*}{ HR (bpm) } & T1 & $81.83 \pm 10.15$ & $72.33 \pm 4.03$ \\
\cline { 2 - 4 } & T2 & $78.00 \pm 9.44$ & $71.83 \pm 5.31$ \\
\cline { 2 - 4 } & T3 & $85.83 \pm 14.16$ & $72.00 \pm 9.34$ \\
\hline \multirow{3}{*}{ SBP(mmHg) } & T1 & $130.83 \pm 18.82$ & $123.00 \pm 13.48$ \\
\cline { 2 - 4 } & T2 & $111.83 \pm 9.09 \dagger$ & $116.83 \pm 6.46$ \\
\cline { 2 - 4 } & T3 & $124.17 \pm 10.21 \ddagger$ & $117.33 \pm 3.39$ \\
\hline \multirow{3}{*}{ DBP(mmHg) } & T1 & $80.00 \pm 6.32$ & $70.83 \pm 8.75$ \\
\cline { 2 - 4 } & T2 & $76.00 \pm 5.10$ & $69.17 \pm 8.13$ \\
\cline { 2 - 4 } & T3 & $83.33 \pm 2.58 \ddagger$ & $71.50 \pm 7.69$ \\
\hline \multirow{3}{*}{ MAP(mmHg) } & T1 & $96.94 \pm 6.95$ & $88.22 \pm 8.49$ \\
\cline { 2 - 4 } & T2 & $87.95 \pm 6.00 \dagger$ & $85.06 \pm 6.88$ \\
\cline { 2 - 4 } & T3 & $96.94 \pm 4.77 \ddagger$ & $86.78 \pm 4.91$ \\
\hline
\end{tabular}

Table 2: Heart rate and blood pressure values at the 3 time points.

$\mathrm{HR}=$ Heart Rate; bpm $=$ beats per minute; $\mathrm{SBP}=$ Systolic Blood Pressure; DBP= Diastolic Blood Pressure; MAP = Mean Arterial Pressure; $\mathrm{mmHg}=$ millimeters of mercury; $\mathrm{T} 1=$ baseline; $\mathrm{T} 2=$ immediately after the end of training; $\mathrm{T} 3=$ after detraining phase; †significant difference between $\mathrm{T} 1$ and $\mathrm{T} 2$; tsignificant difference between $\mathrm{T} 2$ and $\mathrm{T} 3 ; \mathrm{p}<0.05$.
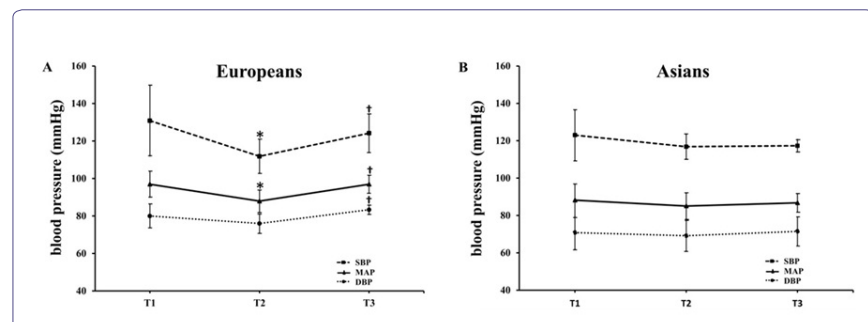

Figure 1: Blood pressure response to Qigong exercise in European (A) and Asian participants (B).

$\mathrm{SBP}=$ Systolic Blood Pressure DBP $=$ Diastolic Blood Pressure $;$ MAP $=$ Mean Arterial Pressure; $\mathrm{mmHg}=$ millimeters of mercury; $\mathrm{T} 1=$ baseline; $\mathrm{T} 2=$ immediately after the end of training; T3 = after detraining phase; *significant difference between baseline and immediately after the end of training; $\uparrow$ significant difference between immediately after the end of training and after detraining phase, $\mathrm{p}<0.05$.

\begin{tabular}{|c|c|c|c|}
\hline Group & T1 (beats/min) & T2 (beats/min) & p \\
\hline Europeans & $118.67 \pm 9.48$ & $112.83 \pm 7.78$ & 0.03 \\
\hline Asians & $113.50 \pm 4.32$ & $101.83 \pm 11.87$ & 0.04 \\
\hline \multicolumn{4}{|c|}{ Table 3: Heart rate at submaximal exercise. } \\
T1 = baseline; T2 $=$ immediately after the end of training. \\
\hline
\end{tabular}

When the data were adjusted for age and BMI, the similar trends were found. Asian participants, there was no significant difference among T1, T2 and T3 for all parameters except submaximal HR decreased significantly after Qigong training $(p=0.04)$. European participants, MAP and submaximal HR decreased significantly after Qigong training $(\mathrm{p}=0.02$ and $\mathrm{p}=0.04$, respectively). SBP tended to decrease after training $(\mathrm{p}=0.06$ for age adjustment and $\mathrm{p}=0.06$ for age and BMI adjustment). After adjusted for age, European participants had higher baseline DBP and baseline MAP than Asian participants $(p=0.02$ and $p=0.04$, respectively), baseline DBP still higher in European participants when data were adjusted for BMI $(\mathrm{p}=0.05)$.

\section{Discussion}

The present study revealed that an 8 weeks of Qigong training significantly improved SBP and MAP in European participants which returned to baseline 12 weeks after Qigong cessation. Only a slight but insignificant lowering of SBP was found in Asians. In addition, submaximal exercise heart rates have significantly improved after Qigong training in both Asian and European participants.

Control of breathing during Qigong training may alter the autonomic nervous system function and contribute to improvement of cardiovascular function at rest and during submaximal exercise as well. The results of the present study are consistent with that reported by Lee et al., who showed that Qigong training significantly reduced blood pressure in hypertensive patients. The authors suggested that Qigong may decrease systemic blood pressure by stabilizing the sympathetic nervous system [4]. In the present study, Qigong training had a more clear effect on SBP and MAP in the European than the Asian participants. These finding is in agreement with previous studies demonstrating that ethnicity may impact differently on the response to exercise training. For instance, Swift et al., reported that African American postmenopausal women had an attenuated increase in cardiorespiratory fitness following exercise training compared with Caucasian American women [11]. Rice et al., suggested that exercise responses/trainability of SBP and HR were associated with genetic factors [16]. On the other hand, Skinner et al., demonstrated that there are no significant differences between blacks and whites in the mean increase in $\mathrm{VO}_{2 \max }\left(\mathrm{ml} / \mathrm{min}\right.$ or $\left.\mathrm{ml} \cdot \mathrm{kg} \mathrm{FFM}^{-1} \cdot \mathrm{min}^{-1}\right)$ with exercise training. The study suggested that race has no significant effect on the response of $\mathrm{VO}_{2 \text { max }}$ to exercise training [17].

The larger decrease of blood pressure after Qigong training in European participants might be due to the somewhat higher baseline systemic blood pressure compared to the Asian participants. Pescatello and Kulikowich demonstrated that participants with the highest baseline blood pressure showed the greatest post-exercise blood pressure reductions [18]. In addition, Melo et al., showed a positive correlation between baseline blood pressure and its reduction after exercise [19]. However, the association between baseline blood pressure and training induced change in blood pressure is not clear. Kiviniemi et al., suggested that baseline sympathetic predominance may predict the larger training induced decrease in DBP indicating that participants with sympathetic predominance may have greater potential to decrease neural sympathetic activity [20]. The present study was conducted in Innsbruck for European group and in Khon Kaen for Asian group. Innsbruck is located at about 600 mabove sea level (with the opportunity to reach easily higher altitudes) while Khon Kaen between 100-200 meters above sea level [21,22]. Living and training at different altitudes may result in differences in physiological response to training. There are several factors influencing blood pressure response to exercise training such as baseline blood pressure, age, genetics, or environmental factor [16,23-25].

In addition, submaximal exercise HRs was significantly decreased after Qigong training in both groups. This finding is well in agreement with previous studies reporting that a five-week aerobic exercise training program reduced HRs response to submaximal workload with no changes in resting HRs [26]. Carter et al., suggested that endurance exercise training decreases submaximal exercise HRs by reducing sympathetic activity to the heart [27]. Thus, even the low intensity training as done by Qigong training is sufficient to reduce HRs to submaximal exercise. 
Citation: Ladawan S, Leelayuwat N, Wannanon P, Klarod K, Menz V, et al. (2017) Cardiovascular Response to Qigong Training in European and Asian Participants. J Altern Complement Integr Med 3: 040.

\section{Strengths and Limitations}

The strength of the present study is resting BP and submaximal HR changes even in this small group. However, some limitations need to be considered. One of the major limitations of the present study is the small sample size, and participants were recruited from only one country for each group. Another limitation is lack of submaximal HR at T3. However, the results found that submaximal HR significantly decreased after Qigong training in both groups.

\section{Conclusion}

8-week Qigong training improved resting BP and submaximal HRs especially in European participants who showed somewhat higher baseline BP and HRs. However, beneficial effects on blood pressure disappeared after 12 weeks of exercise cessation. Therefore, training continuation is of essential importance. Further research is required to examine whether our SBP findings in response to Qigong training were due to higher baseline blood pressure, sample bias (migration) or ethnicity differences.

\section{Acknowledgment}

This study was supported by Exercise and Sport Sciences Development and Research Group, Department of Physiology, Faculty of Medicine, Khon Kaen University, Thailand, and a grant from The ASEAN European Academic University Network (ASEA-UNINET).

\section{References}

1. Chow YW, Tsang HW (2007) Biopsychosocial effects of qigong as a mindful exercise for people with anxiety disorders: a speculative review. J Altern Complement Med 13: 831-839.

2. Bernardi L, Porta C, Gabutti A, Spicuzza L, Sleight P (2001) Modulatory effects of respiration. Auton Neurosci 90: 47-56.

3. Lee MS, Lee MS, Kim HJ, Choi ES (2004) Effects of qigong on blood pressure, high-density lipoprotein cholesterol and other lipid levels in essential hypertension patients. Int J Neurosci 114: 777-786.

4. Lee MS, Lee MS, Kim HJ, Moon SR (2003) Qigong reduced blood pressure and catecholamine levels of patients with essential hypertension. Int J Neurosci 113: 1691-1701.

5. Lee MS, Lim HJ, Lee MS (2004) Impact of qigong exercise on self-efficacy and other cognitive perceptual variables in patients with essential hypertension. J Altern Complement Med 10: 675-680.

6. Chang MY (2015) Qigong Effects on Heart Rate Variability and Peripheral Vasomotor Responses. West J Nurs Res 37: 1383-1403.

7. http://taichi18.com

8. Donnelly PM, Yang TS, Peat JK, Woolcock AJ (1991) What factors explain racial differences in lung volumes? Eur Respir J 4: 829-838.

9. Korotzer B, Ong S, Hansen JE (2000) Ethnic differences in pulmonary function in healthy nonsmoking Asian-Americans and European-Americans. Am J Respir Crit Care Med 161: 1101-1108.
10. Bouchard C, Rankinen T (2001) Individual differences in response to regular physical activity. Med Sci Sports Exerc 33: 446-451.

11. Swift DL, Johannsen NM, Lavie CJ, Earnest CP, Johnson WD, et al. (2013) Racial differences in the response of cardiorespiratory fitness to aerobic exercise training in Caucasian and African American postmenopausal women. J Appl Physiol 114: 1375-1382.

12. Chao YF, Chen SY, Lan C, Lai JS (2002) The cardiorespiratory response and energy expenditure of Tai-Chi-Qui-Gong. Am J Chin Med 30: 451461 .

13. Kjos V, Etnier JL (2006) Pilot study comparing physical and psychological responses in medical qigong and walking. J Aging Phys Act 14: 241-253.

14. Lee MS, Lee MS, Choi ES, Chung HT (2003) Effects of Qigong on blood pressure, blood pressure determinants and ventilatory function in middle-aged patients with essential hypertension. Am J Chin Med 31: 489497.

15. http://www.chirontaichi.co.uk/downloads/taiji_qigong_18_movements. pdf

16. Rice T, An P, Gagnon J, Leon AS, Skinner JS, et al. (2002) Heritability of $\mathrm{HR}$ and BP response to exercise training in the HERITAGE Family Study. Med Sci Sports Exerc 34: 972-979.

17. Skinner JS, Jaskolski A, Jaskolska A, Krasnoff J, Gagnon J, et al. (2001) Age, sex, race, initial fitness, and response to training: the HERITAGE Family Study. J Appl Physiol 90: 1770-1776.

18. Pescatello LS, Kulikowich JM (2001) The aftereffects of dynamic exercise on ambulatory blood pressure. Med Sci Sports Exerc 33: 1855-1861.

19. Melo CM, Alencar Filho AC, Tinucci T, Mion D Jr, Forjaz CL (2006) Postexercise hypotension induced by low-intensity resistance exercise in hypertensive women receiving captopril. Blood Press Monit 11: 183-189.

20. Kiviniemi AM, Hautala AJ, Karjalainen JJ, Piira OP, Lepojärvi S, et al. (2014) Acute post-exercise change in blood pressure and exercise training response in patients with coronary artery disease. Front Physiol 5: 526.

21. https://www.innsbruck.info/en/innsbruck-city.html

22. Kantawateera K, Naipinit A, Sakolnakorn TPN, Churngchow C, Kroeksakul P (2013) A SWOT Analysis of Tourism Development in Khon Kaen, Thailand. Asian Soc Sci 9: 226-231.

23. Kelley GA, Kelley KA, Tran ZV (2001) Aerobic exercise and resting blood pressure: a meta-analytic review of randomized, controlled trials. Prev Cardiol 4: 73-80.

24. Ishikawa K, Ohta T, Zhang J, Hashimoto S, Tanaka H (1999) Influence of age and gender on exercise training-induced blood pressure reduction in systemic hypertension. Am J Cardiol 84: 192-196.

25. Park H-Y, Lim K (2017) The effects of aerobic exercise at hypoxic condition during 6 weeks on body composition, blood pressure, arterial stiffness, and blood lipid Level in obese women. Int J Sports Sci Med 1: 1-5.

26. Pigozzi F, Alabiso A, Parisi A, Di Salvo V, Di Luigi L, et al. (2001) Effects of aerobic exercise training on $24 \mathrm{hr}$ profile of heart rate variability in female athletes. J Sports Med Phys Fitness 41: 101-107.

27. Carter JB, Banister EW, Blaber AP (2003) Effect of endurance exercise on autonomic control of heart rate. Sports Med 33: 33-46. 
Citation: Ladawan S, Leelayuwat N, Wannanon P, Klarod K, Menz V, et al. (2017) Cardiovascular Response to Qigong Training in European and Asian Participants. J Altern Complement Integr Med 3: 040.

- Page 5 of $4 \cdot$ 